\title{
ASSOCIAÇÃO ENTRE OPHIONEREIS RETICULATA (SAY) (OPHIUROIDEA, OPHIONEREIDIDAE) E MALMGRENIELLA VARIEGATA (TREADWELL) (POLYCHAETA, POLYNOIDAE) DAS PRAIAS DE SALVADOR, BAHIA, BRASIL
}

\author{
Lêda Maria de Santa-Isabel ${ }^{1}$ \\ Walter R. Pinto Cerqueira ${ }^{1,2}$ \\ Orane Falcão de Souza Alves ${ }^{1}$
}

\begin{abstract}
ASSOCIATION BETWEen Ophionereis RETICUlata (SAY) (OpHIUROIDEA, Ophionereididae) and Malmgreniella Variegata (TREAdWell) (Polychaeta, Polynoidae) from Salvador beAches, Bahia, Brazil. The occurrence and distribution records of the association between Ophionereis reticulata (Say) and Malmgreniella variegata (Treadwell) in Northeastern Brazil, and the redescription of M. variegata, are included.

KEY WORDS. Ophiuroidea, Ophionereididae, Malmgreniella variegata, Polychaeta, Polynoidae
\end{abstract}

Dentre os trabalhos relativos à associação entre Ophionereis reticulata (Say, 1825) e polinoídeos comensais, destacam-se as publicações de MillotT (1953), Weston (1984) e PetTiBOne (1993). Porém, no que diz respeito à costa brasileira, não se tem nenhuma referência a respeito de tal relação.

Durante coletas realizadas para levantamento de equinodermos das praias do litoral de Salvador, observou-se, por acaso, a associação entre $O$. reticulata e Malmgreniella variegata (Treadwell, 1917). O. reticulata tem uma larga distribuição no litoral brasileiro, enquanto que a distribuição de $M$. variegata é citada apenas para a região sul e sudeste, conforme os trabalhos de AMARAL \& NONATO (1981) e LANA (1984).

O presente trabalho tem como objetivos não somente registrar a ocorrência e a distribuição da associação entre as duas espécies acima citadas para o Nordeste do Brasil , como também a redescrição de $M$. variegata.

\section{MATERIAL E MÉTODOS}

Cinco exemplares de ofiúros associados a poliquetas foram obtidos manualmente, em zona intertidal, revirando-se fragmentos de rochas, durante as coletas para a realização de um inventário dos equinodermos das Praias de Salvador. Os animais coletados foram acondicionados em baldes contendo água do mar e conduzidos ao laboratório.

1) Departamento de Zoologia, Instituto de Biologia, Universidade Federal da Bahia. Campus Universitário de Ondina, 40170-210 Salvador, Bahia, Brasil.

2) Bolsista do CNPq. 
Em laboratório, os indivíduos foram colocados em aquário contendo fundo com cascalho e água do mar, devidamente aerado, para observação comportamental e em seguida anestesiados com Cloreto de Magnésio 7,5\%, fixados em formol neutro $4 \%$ e conservados em álcool glicerinado (Ophiuroidea) e álcool 70\% (Polychaeta).

O material foi posteriormente identificado de acordo com TOMMASI (1970) e Thomas (1973) (Ophiuroidea), e Fauchald (1977) e Pettibone (1993) (Polychaeta), e encontra-se depositado na coleção do Departamento de Zoologia do Instituto de Biologia (Universidade Federal da Bahia).

\section{Polynoidae}

\section{Malmgreniella Hartman, 1967}

\section{Malmgreniella variegata (Treadwell, 1917) \\ Figs 1-11}

Harmothoe variegata Treadwell, 1917: 260, pl. 1: figs 17, 18; pl. 2: figs 1-3.

Harmothoe lunulata; Millott, 1953: 96, figs 1, 2 [não Delle Chiaje, 1830]. - Day, 1967:71, fig. 1.10p-t. - Amaral \& Nonato, 1981: 22. -Lana, 1984: 23.

Malmgreniella variegata; Pettibone, 1993: 45, fig. 30.

Diagnose. Encontrado em O. reticulata. Espécime com $16 \mathrm{~mm}$ de comprimento, $3 \mathrm{~mm}$ de largura, 36 segmentos. Corpo elongado, achatado. Escamas com marcas pretas ou marrons; na parte dorsal, os $25^{\circ}$ e $28^{\circ}$ setígeros apresentam uma pigmentação escura na base dos elitróforos (Fig. 2); longitudinalmente, na parte ventral, encontram-se manchas pigmentares médio-ventrais e nas bases laterais dos parapódios (Fig. 3) e, anteriormente, na parte superior, médio, lateral e na margem posterior da boca. Quinze pares de escamas cobrindo o dorso exceto poucos segmentos posteriores. Escama oval a sub-reniforme, sem papilas, com grupos de microtúbulos próximos às margens anteriores (ausentes na primeira e em algumas escamas posteriores); pigmentação mais densa em algumas áreas, consistindo de uma larga área semelhante a um funil na metade posterior da escama, irradiando para a região de fixação do elitróforo, continuando como uma faixa estreita ao longo da borda látero-posterior; uma faixa estreita transversa completa o ciclo (Figs 4-6). Prostômio bilobado com lobos anteriores truncados ou sub-triangulares, com dois pares de manchas ocelares bastante pequenas e separadas; ceratóforo da antena mediana robusto, com estilete longo e totalmente pigmentado, ceratóforo das antenas laterais inserido término-ventralmente e com estiletes curtos; palpos robustos, afilados com fileiras longitudinais de pequenas papilas; cada base dos tentáculos com pequenos lobos aciculares no lado interno, sem setas, e um par de cirros tentaculares dorsal e um ventral similar às antenas laterais medianas; todos os apêndices variadamente pigmentados (Fig. 1). Segmento 2 com o primeiro par de elitróforos e parapódios birremes. Notopódio menor que o neuropódio; notopódio arrendondado com lobo acicular no lado inferior; neuropódio mais largo com lobo acicular presetal sub-cônico mais longo, digitiforme. Cirro dorsal, com cirróforo 

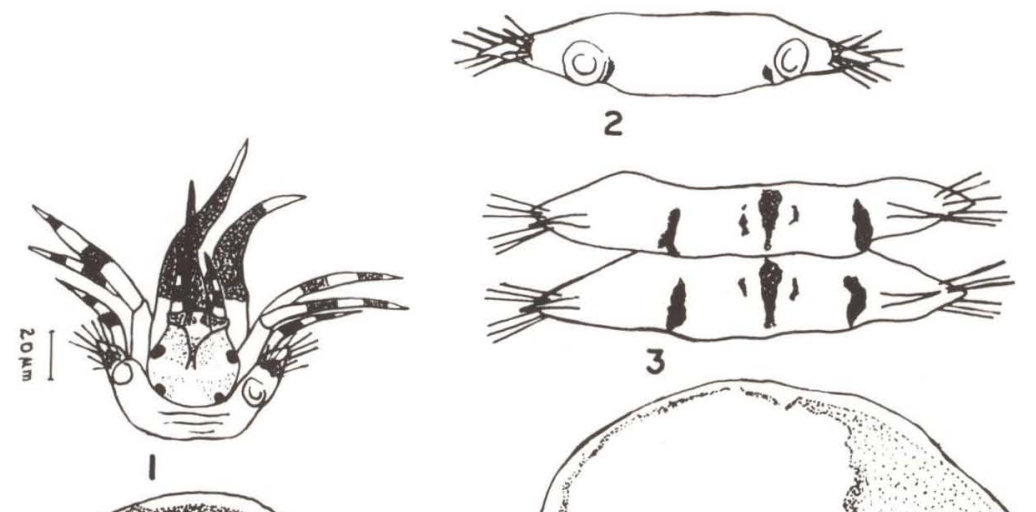

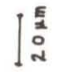

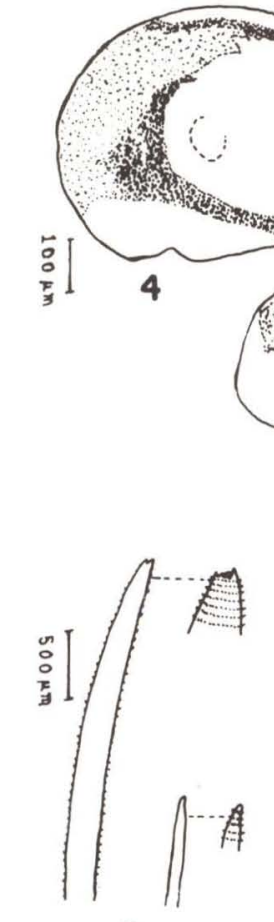

9

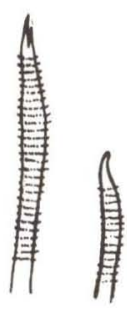

$10 \quad 11$
()

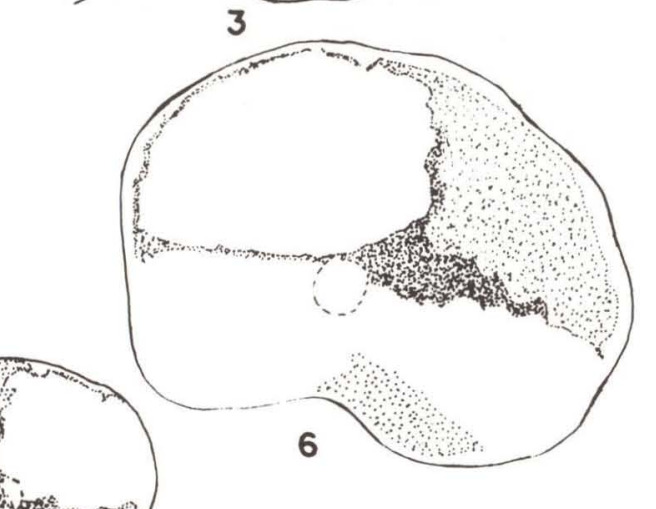

50

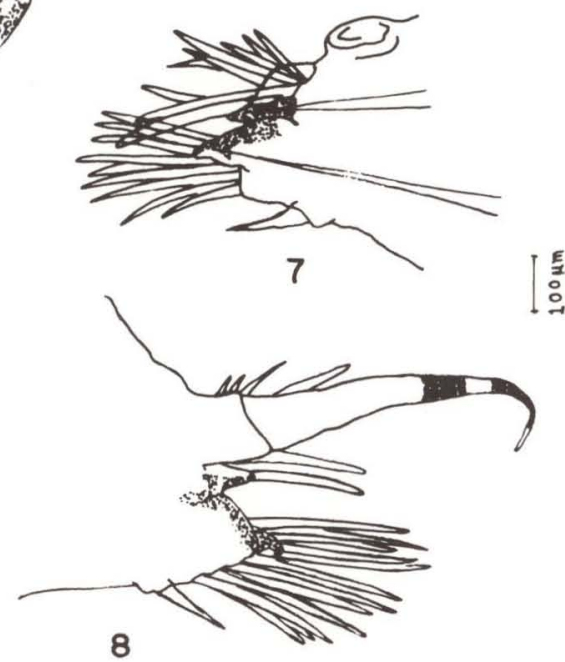

Figs 1-11. Malmgreniella variegata. (1) Extremidade anterior; (2) parte dorsal do segmento 28 mostrando pigmentação na base do elitróforo; (3) vista ventral dos segmentos 21 e 22 mostrando pigmentação; (4) primeira escama esquerda do segmento 2; (5) segunda escama esquerda do segmento 4, destacando os microtúbulos; (6) sexta escama direita do segmento 11; (7) parapódio elitrigero direito, vista anterior, com detalhe das acículas; (8) parapódio cirrigero direito, vista posterior; (9) notosetas longa e curta, detalhando as extremidades; (10) neuroseta mediana; (11) neuroseta menor. Escalas $=500 \mu \mathrm{m}$ para figuras $4-8$ e $100 \mu \mathrm{m}$ para figuras 9-11. 
cilíndrico e estilete alongado ultrapassando as neurosetas, com duas faixas escuras próximas da extremidade; cirro ventral curto, afilado e sem pigmentação (Figs 7-8). Notosetas numerosas formando feixes mais robustos que as neurosetas, com numerosas fileiras espinhosas, notosetas curtas com extremidade afilada, e longas com extremidade com cerca de quatro dentes. Neurosetas formando um leque com numerosas e fracas fileiras espinhosas, todas com extremidade distal ligeiramente curvada, exceto algumas neurosetas mais curtas, com dentes secundários (Figs 9-11). Pigídio com ânus entre o último par de pequenos parapódios, com um par de longos cirros anais com pigmentação escura.

Os polinoídeos, associados à $O$. reticulata, tratados como Harmothoe lunulata na publicação de MiLlotT (1953), assim como os não associados, citados nos trabalhos de DAY (1967), AMARAL \& NONATO (1981) e LANA (1984), são considerados por PETTIBONE (1993) como Malmgreniella variegata.

Biologia. Cinco espécimes de $M$. variegata foram encontradas em areia sob rochas submersas, na praia de Ondina, associados a $O$. reticulata. Em aquário, durante observação comportamental, percebeu-se que, em todos os casos, o polinoídeo permanecia praticamente imóvel sobre a face aboral do disco do ofiúro, supondo-se assim, tratar-se de uma simbiose. Após a difícil tarefa de separar os animais, pois o polinoídeo agarrava-se cada vez mais ao ofiúro, os indivíduos foram diexados próximos um do outro. O poliqueta migrava ou para a face oral ou para os braços do ofiúro e em seguida retornava ao disco aboral. Uma vez totalmente separados, o polinoídeo caminhava ativamente ao redor do recipiente, onde se encontrava, como se procurasse o ofiúro. Este último, após a primeira separação, estendia os braços em direção ao poliqueta permitindo a fixação do mesmo.

Apesar de poucas observações e da falta de análises comportamentais, o que limitou bastante a conclusão do trabalho, no que diz respeito às adaptações fisiológicas, pode-se perceber que há uma especificidade em relação à associação, uma vez que, em habitat natural, apesar de existirem outras espécies de ofiúros no local de coleta, apenas o O. reticulata encontrava-se associado a $M$. variegata. Contudo, conforme HENDLER \& MEYER (1982), tanto O. reticulata quanto O. annulata, que têm padrões de coloração e pigmentações semelhantes, se associam à $M$. variegata. Além disso, comparando-se o comportamento dos indivíduos após a separação, verificou-se também um maior nível de dependência do polinoídeo em relação ao ofiúro, uma vez que o poliqueta agarrava-se ao seu companheiro tentando impedir a separação.

Quanto as adaptações morfológicas o polinoídeo apresentou também um grau superior ao do ofiúro.

Em relação à cor, as escamas são brancas com um padrão preto bem definido e repetitivo, igualando-se ao padrão de coloração do ofiúro (Fig. 12). Os tipos de setas são semelhantes a ganchos, apropriados para fixação nos espinhos dos braços do ofiúro, fato também registrado por MiLLOTT (1953).

Em geral os polinoídeos apresentam associações com outros invertebrados, inclusive com outras espécies de ofiúros (Ophiophragmus cubanus H.L. Clark, 1918 e O. pulcher H.L. Clark) (PETTIBONE 1993), cujas relações são consideradas como Comensalismo por Millott (1953), WESTON (1984) e PETTIBONE (1993). 
Distribuição. Noroeste do Oceano Atlântico, Flórida; Golfo do México; Mar do Caribe; Leste do Oceano Pacífico, Golfo do Panamá; Sul do Oceano Atlântico, Nordeste do Brasil.

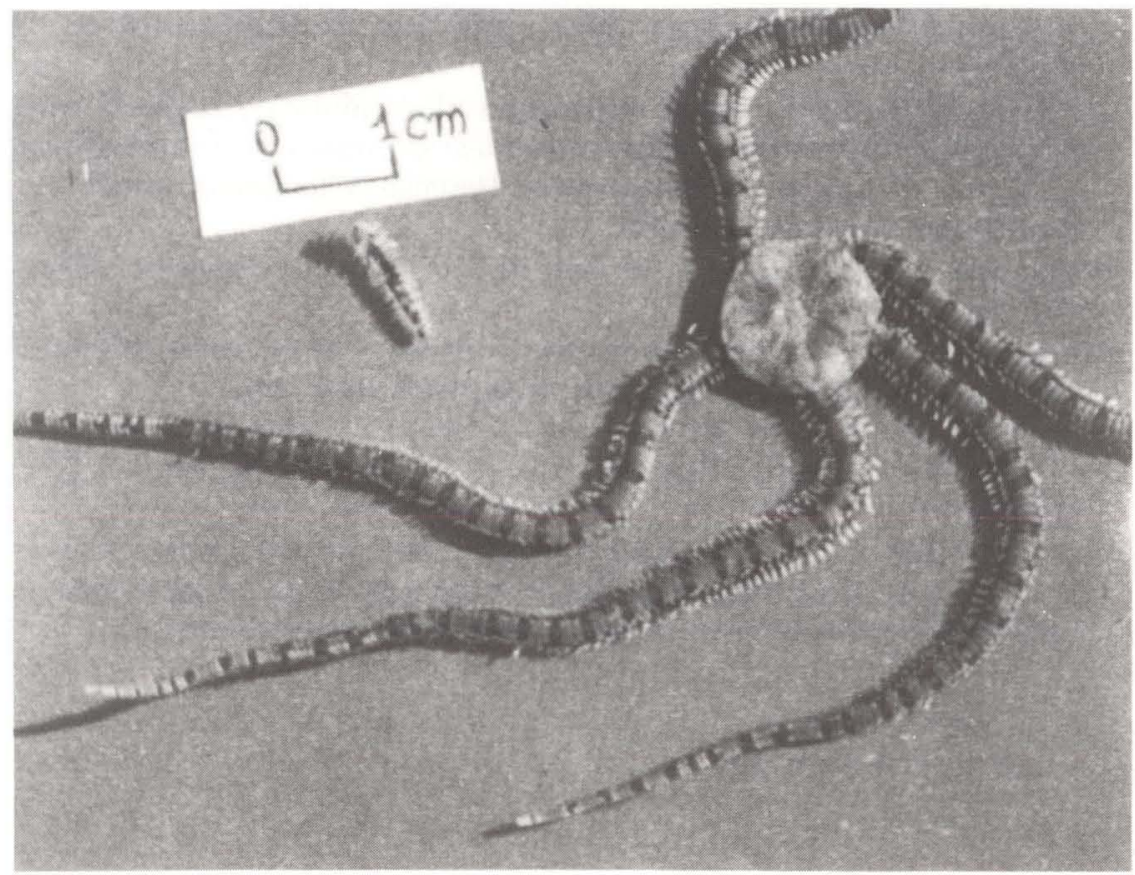

Fig. 12. Malmgreniella variegata com Ophionereis reticulata.

AGRADECIMENTOS. Ao Dr. Paulo Lana (Centro de Estudos do Mar, Universidade Federal do Paraná) pela confirmação da espécie de poliqueta.

\section{REFERÊNCIAS BIBLIOGRÁFICAS}

AMARAL, A.C.Z.\& E.F. NONATO. 1981. Anelídeos poliquetas da costa brasileira. Características e chave para famílias. Brasília, CNPq Coordenação Editorial, vol. $1 / 2,47 \mathrm{p}$.

DAY, J. 1967. A monograph on the polychaeta of Southern Africa. Part 1: Errantia. Brit. Mus. Nat. Hist. Publ. 656: 1-458.

Fauchald, K. 1977. The Polychaete worms. Definitions and keys to the orders, families and genera. Nat. Hist. Mus. Los Angeles Cty., Sci. Ser. 28: 1-188.

HendLer, G. \& D.L. MEYER. 1982. An Association of a Polychaete, Branchiosyllis exilis with an Ophiuroid, Ophiocoma echinata, in Panama. Bull. Mar. Sci. 32 (3): 736-744. 
LANA, P. 1984. Anelídeos poliquetas errantes do litoral do Estado do Paraná. Tese de doutorado, não publicada, Instituto Oceanográfico, Universidade de São Paulo, São Paulo, 275p.

MillotT, N. 1953. A remarkable association between Ophionereis reticulata (Say) and Harmothoe lunulata (Delle Chiaje ). Bull. Mar. Sci. Gulf Caribb. 3 (2): 96-99.

Pettibone, M.H. 1993. Scaled polychaetes (Polynoidae) associated with ophiuroids and other invertebrates and review of species referred to Malmgrenia McIntosh and replaced by Malmgreniella Hartman, with descriptions of new taxa. Smiths. Contrib. Zool. 538: 1-92.

Thomas, L.P. 1973. Western Atlantic brittlestars of the genus Ophionereis. Bull. Mar. Sci. 23 (3): 585-599.

Tommasi, L.R. 1970. Os ofiuróides recentes do Brasil e regiões vizinhas. Contr. Inst. Oceanogr. Univ. S. Paulo, Ser. Ocean. Biol. 20: 1-146.

TREAdWELL, A.L. 1917. Polychaetous annelids from Florida, Porto Rico, Bermuda and the Bahamas. Papers Dep. Mar. Biol. Carnegie Inst., Washington, 251: 255-272.

Weston, D. 1984. Family Polynoidae Malmgren, 1867. In: J.M. UeBelacker \& P.G. JoHnson (Eds). Taxonomic Guide to the Polychaetes of the Northern Gulf of Mexico 3 (21): 1-39. Alabama, Barry Vittor and Associates.

Recebido em 30.V.1995; aceito em 02.VIII.1996. 